\title{
Aging and Inhibitory Control of Action: Cortico-Subthalamic Connection Strength Predicts Stopping Performance
}

\author{
James P. Coxon, Annouchka Van Impe, Nicole Wenderoth, and Stephan P. Swinnen \\ Movement Control and Neuroplasticity Research Group, Katholieke Universiteit Leuven, 3001 Leuven, Belgium
}

\begin{abstract}
Diffusion weighted imaging (DWI) studies in humans have shown that seniors exhibit reduced white matter integrity compared with young adults, with the most pronounced change occurring in frontal white matter. It is generally assumed that this structural deterioration underlies inhibitory control deficits in old age, but specific evidence from a structural neuroscience perspective is lacking. Cognitive action control is thought to rely on an interconnected network consisting of right inferior frontal cortex ( $\mathrm{r}$-IFC), pre-supplementary motor area (preSMA), and the subthalamic nucleus (STN). Here we performed probabilistic DWI tractography to delineate this cognitive control network and had the same individuals (20 young, 20 older adults) perform a task probing both response inhibition and action reprogramming. We hypothesized that structural integrity (fractional anisotropy) and connection strength within this network would be predictive of individual and age-related differences in task performance. We show that the integrity of r-IFC white matter is an ageindependent predictor of stop-signal reaction time (SSRT). We further provide evidence that the integrity of white matter projecting to STN predicts both outright stopping (SSRT) and transient braking of response initiation to buy time for action reprogramming (stopping interference effects). These associations remain even after controlling for Go task performance, demonstrating specificity to the Stop component of this task. Finally, a multiple regression analysis reveals bilateral preSMA-STN tract strength as a significant predictor of SSRT in older adults. Our data link age-related decline in inhibitory control with structural decline of STN projections.
\end{abstract}

\section{Introduction}

One of the prevailing theories in cognitive aging is that inhibitory processing is compromised. This fundamental deficit is thought to influence cognitive performance in a variety of domains (Hasher and Zacks, 1988; Lustig et al., 2007). The neural process underlying inhibition of behavior can take different forms (Munakata et al., 2011), reflecting the many and varied ways in which the term is used (Aron, 2007). However, it is widely accepted that an inhibitory process can countermand an initiated motoric response. The neuroanatomical basis of this form of inhibition is thought to rely critically on the subthalamic nucleus (STN) (Aron and Poldrack, 2006). Importantly, the efficiency of motor inhibition can be measured by calculating stop-signal reaction time (SSRT), which is known to deteriorate with old age (Williams et al., 1999; Bedard et al., 2002). Although it has been assumed that age-related inhibitory deficits stem from reduced frontal lobe integrity (Kramer et al., 1994), this remains to be shown empirically.

Brain white matter integrity declines in old age and is most pronounced in anterior brain regions (Sullivan and Pfefferbaum,

Received Dec. 21, 2011; revised April 22, 2012; accepted May 1, 2012.

Author contributions: J.P.C., N.W., and S.P.S. designed research;J.P.C. performed research;J.P.C., A.V.I., and N.W. analyzed data; J.P.C., A.V.I., N.W., and S.P.S. wrote the paper.

This work was supported by Katholieke Universiteit Research Fund Grant 0T/11/071, Flanders Fund for Scientific Research Grants G0483.10 and G0721.12, and Belgian Federal Government Inter-University Attraction Poles Program Grant P6/29. J.P.C. is supported by Flanders Fund for Scientific Research Postdoctoral Fellowship 1224010N.

Correspondence should be addressed to Dr. James P. Coxon, Movement Control and Neuroplasticity Research Group, Group Biomedical Sciences, Katholieke Universiteit Leuven, Tervuurse Vest 101, 3001 Leuven, Belgium. E-mail: j.coxon@auckland.ac.nz.

DOI:10.1523/JNEUROSCI.6360-11.2012

Copyright $\odot 2012$ the authors $\quad 0270-6474 / 12 / 328401-12 \$ 15.00 / 0$
2006). Concomitant changes occur in gray matter (Fjell et al., 2009) and dopaminergic neurotransmission (Backman et al., 2010). Together, these changes conspicuously parallel reduced processing speed, increased performance variability, and general cognitive decline (Hedden and Gabrieli, 2004). Several studies have explored age-dependent relationships between white matter integrity and composite measures of executive function (Kennedy and Raz, 2009; Vernooij et al., 2009; Zahr et al., 2009); however, composite $z$-scores exclude inference about the neural circuitry supporting a particular process. Executive function encompasses, but is not synonymous with, cognitive control of action, i.e., the rapid inhibition and adjustment of behavior when expectations are violated. It remains untested whether inhibitory deficits in older age are related to frontal white matter in general or to specific tracts critical for task performance (Johansen-Berg, 2010).

Right inferior frontal cortex ( $\mathrm{r}$-IFC) and pre-supplementary motor area (preSMA) are frequently identified as important for a range of tasks requiring response inhibition and switching from automatic to controlled behavior (Aron and Poldrack, 2006; Isoda and Hikosaka, 2007; Chikazoe, 2010; Sharp et al., 2010; Zandbelt and Vink, 2010). These areas are thought to enact control via the basal ganglia, in particular STN (Aron and Poldrack, 2006; Frank, 2006; Isoda and Hikosaka, 2008; Fleming et al., 2010; Hikosaka and Isoda, 2010) to which they are directly connected (Aron et al., 2007). White matter integrity underlying r-IFC and r-preSMA predicts improvement in SSRT during a child's brain development (Madsen et al., 2010). Here we focus on the contribution of projections to STN and test whether $r$ IFC-STN and bilateral preSMA-STN white matter tracts predict 

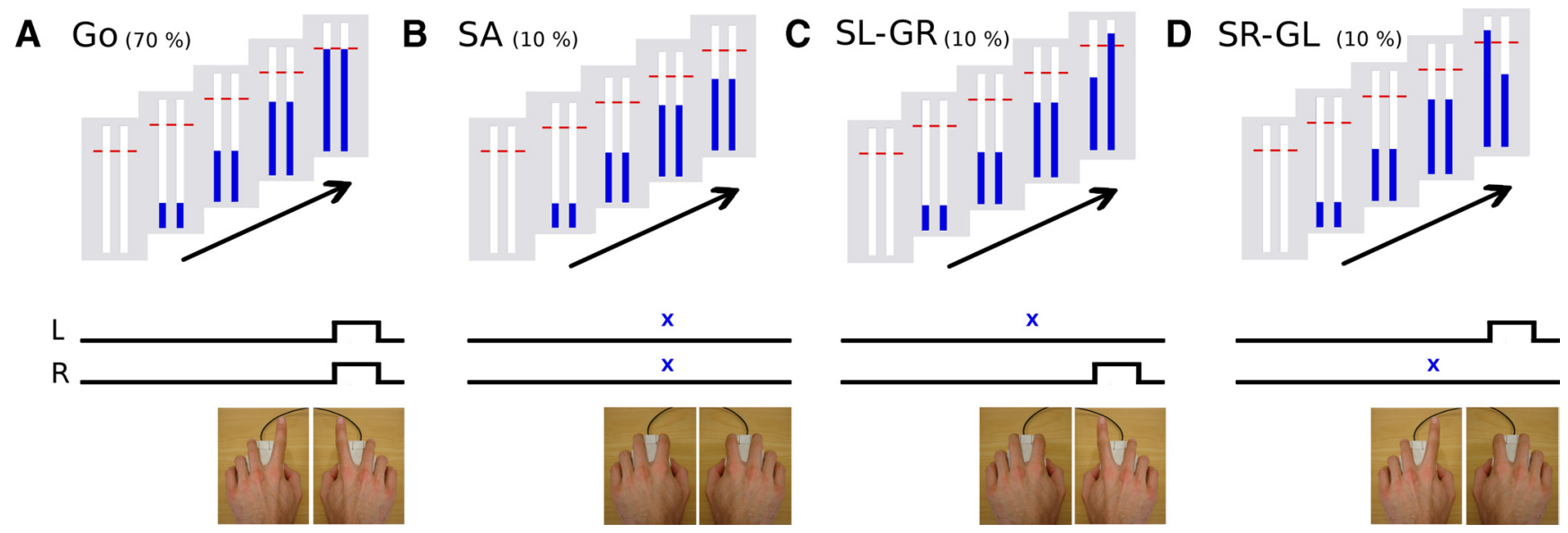

Figure 1. On every trial, participants prepared to simultaneously extend their left $(\mathrm{L})$ and right $(\mathrm{R})$ index fingers to interrupt two continuously moving indicators at a target, $800 \mathrm{~ms}$ from onset. $A, G 0,0 n$ most trials, participants were required to respond as accurately as possible. $B, S A$, When both indicators stopped automatically $(X)$, the successful response was to prevent any movement. $C, D, S L-G R$, When only the left indicator stopped automatically, the successful response was to prevent movement of the left hand while still responding with the other hand and vice versa for SR-GL.

cognitive action control in the context of healthy aging. We outlined STN manually, performed probabilistic tractography, and extracted measures of tract strength and integrity. We hypothesized that connection strength and integrity within this anatomical model of inhibitory control would predict individual and age-related differences in SSRT and interference effects that manifest when both inhibition and action reprogramming is required.

\section{Materials and Methods}

Participants

Twenty old (mean age, 68.7 years; range, $62-81$ years; 9 men) and 20 young (mean age, 25.0 years; range, $20-31$ years; 9 men) adults with normal or corrected-to-normal vision participated in the experiment. All were right handed (laterality quotient: Old, mean of 91.0; Young, mean of 88.5). Participants were within normal limits of general cognitive function as tested by the Mini-Mental State Examination (Old $\geq 27$ of 30 , Young $\geq 29$ of 30). The older participants were independent, community dwelling, and had a similar amount of formal education (Old: mean, 16.6 years; Young: mean, 18.1 years; $p=0.16$ ). The local ethics committee approved the procedure, and all participants gave written, informed consent.

\section{Movement prevention task and experimental procedure}

Participants performed a stop-signal task that probes both global and more selective aspects of response inhibition (Coxon et al., 2007; see also Aron and Verbruggen, 2008). The task provides specific measures reflecting the time needed to overcome an initiated action and the additional time needed for action reprogramming. Such measures are considered sensitive markers of predominantly top-down action control in the aging brain.

Participants were instructed to rest their left and right hands on modified computer mice. Custom software generated a visual display consisting of two vertical indicators that moved from the bottom upward at an equal and constant rate on each trial. A horizontal red target line was situated $800 \mathrm{~ms}$ from onset (Fig. 1). All participants received a standardized description of the task, during which task conditions (Go, Stop All, and Stop Selective) were sequentially introduced. They were instructed to look at the red line throughout the experiment and to interrupt both indicators at this target by extending their index fingers (referred to as Go trials). It was emphasized that Go trials should be performed as accurately and consistently as possible. Next it was explained that the indicator(s) would sometimes stop automatically before reaching the target line and that, when this happened, they should try to prevent moving the corresponding hand (referred to as Stop trials). Three possibilities were introduced: (1) Stop All (SA), in which both indicators stopped at the same time before the target; (2) Stop Left (SL-GR), in which the left indicator stopped before the target; and (3) Stop Right (SR-GL), in which the right indicator stopped before the target. SL and SR refer to the hand to be prevented from moving (i.e., Stop), whereas GR and GL refers to the hand for which action was still required (i.e., Go). Stopping could be made less/more difficult by altering the time that the indicator stopped before the target (Coxon et al., 2006, 2007). Participants were instructed not to worry if they sometimes responded anyway, because the experiment was designed to determine how much time they needed to prevent movement. A short demonstration by the experimenter followed this description.

A Go trial consisted of a black warning rectangle for $500 \mathrm{~ms}$, after which both indicators started to fill upward (Fig. 1A). When the subject simultaneously extended both fingers to interrupt the indicators, the software recorded the response time relative to the target (in milliseconds) for each response switch. One second after trial onset, the indicators were reset to empty. Stop trials were identical up until the point at which either one, or both, indicators stopped automatically before the target (Fig. $1 B-D$ ). In the case in which only one indicator stopped, the other indicator continued until the subject responded with the corresponding hand (for example, SL-GR requires the subject to Go with the right hand).

Participants practiced the task, first performing Go trials + SA, then Go trials + SL-GR and SR-GL, then all possible conditions combined ( $\sim 10 \mathrm{~min})$. They then performed a block consisting only of Go trials (40 trials), followed by five blocks of 100 trials each. Each block consisted of Go (70\%), SA (10\%), SL-GR (10\%), and SR-GL (10\%) trials, presented in a random order. For the Stop conditions, there were five stop times, also randomized. In total, there were 350 Go trials and 50 trials per Stop condition (10 trials per stop time). For SA, both indicators stopped automatically $270,230,190,150$, and 110 ms before the target. Because $\sim 30$ ms more time is required on selective trials (Coxon et al., 2007), the indicator stopped automatically $300,260,220,180$, and 140 ms before the target for SL-GR and SR-GL.

\section{Behavioral data analysis}

For each Stop condition, the probability of responding, $P$ (respond), was calculated at each stop time, with $P$ (respond $)=0$, indicating consistently successful inhibition and $P$ (respond $)=1$ indicating that movement prevention was impossible. The critical stop time $\left(\mathrm{ST}_{\text {crit }}\right)$ was determined using weighted linear interpolation to find $P($ respond $)=0.5$, using the formula $\mathrm{ST}_{\text {crit }}=t 1+[(t 2-t 1) \times(0.5-p 1) /(p 2-p 1)]$, where $t 1$ is the first stop time with an inhibition probability of $<0.5, t 2$ is the first stop time with an inhibition probability of $>0.5$, and $p 1$ and $p 2$ are the inhibition probabilities at $t 1$ and $t 2$, respectively. The primary dependent variable, SSRT, was calculated as the difference between $\mathrm{ST}_{\text {crit }}$ and mean Go trial response time, averaged across hands. We verified our approach 
A

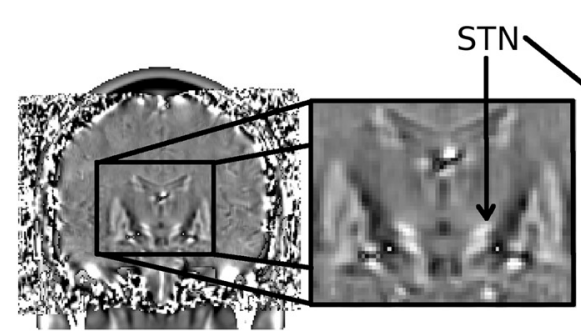

$y-12$

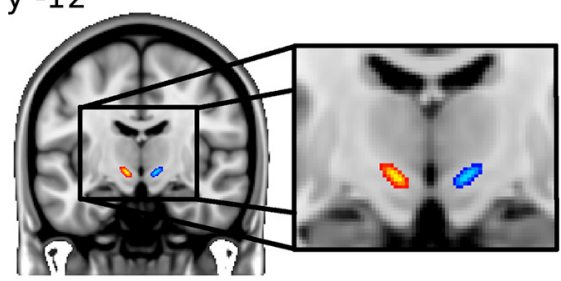

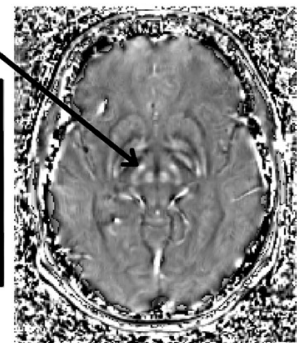

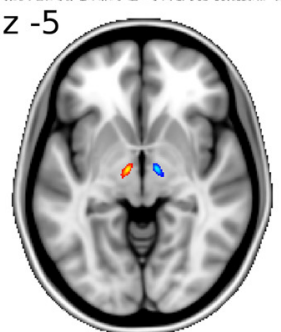

$y+14$

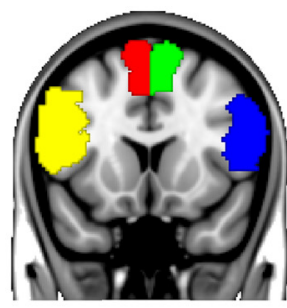

$x-9$

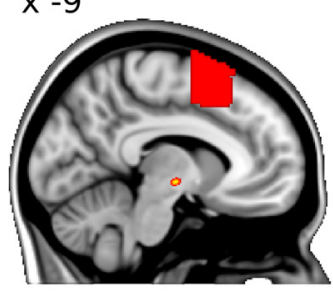

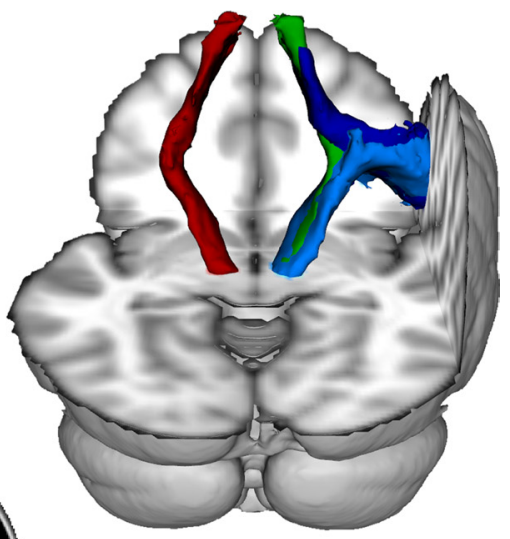

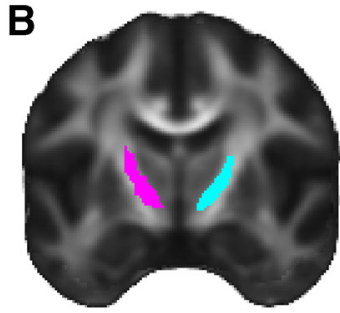

y -8

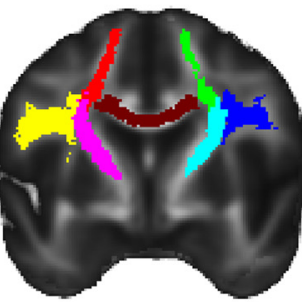

y 0

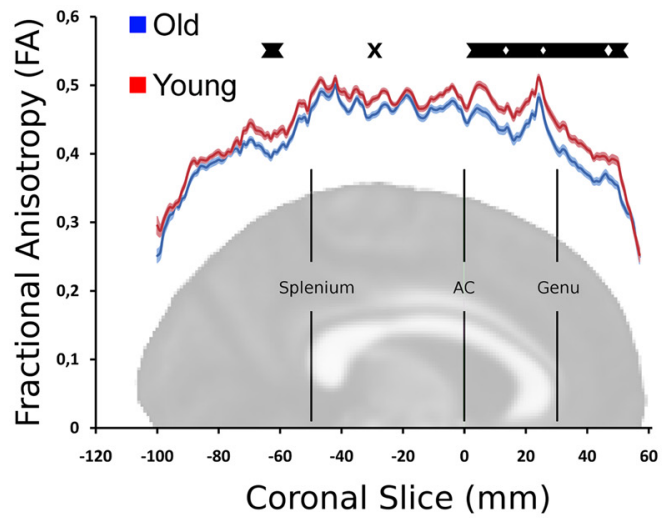

C

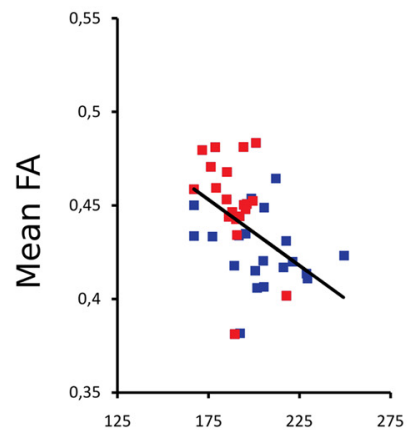

SSRT (ms)

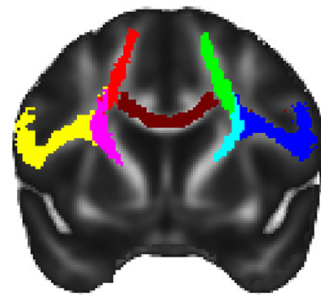

$y+6$

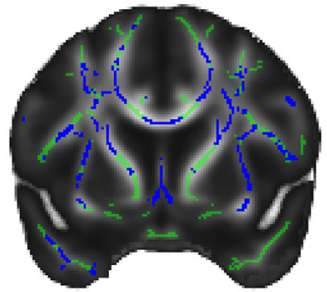

$$
\mathrm{x}-18
$$

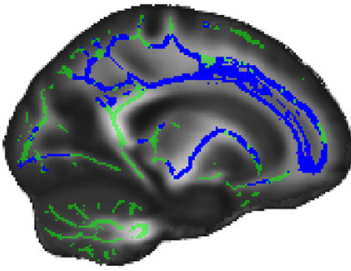

I-preSMA \& I-STN
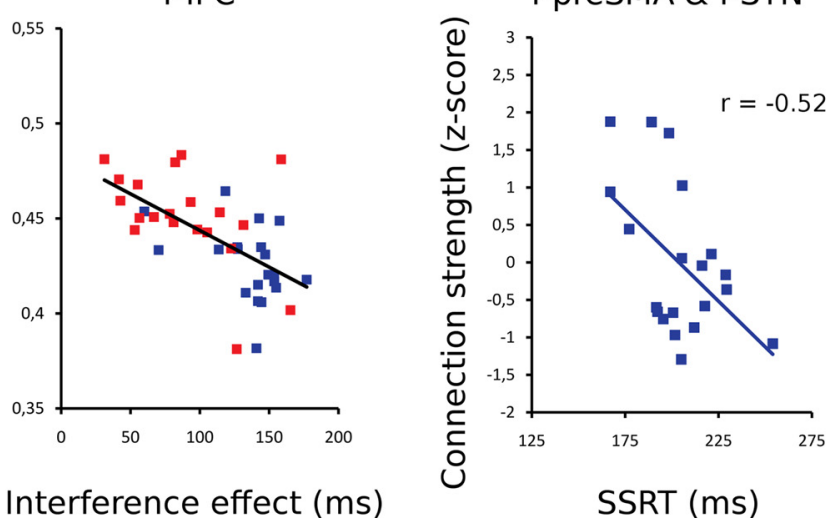

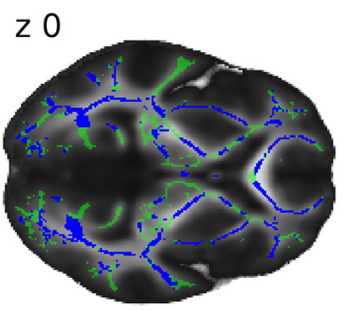

r-preSMA \& r-STN

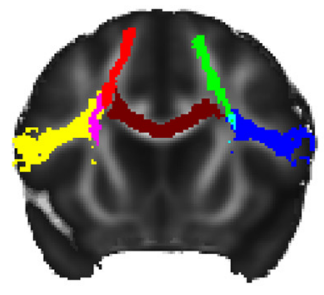

$y+14$

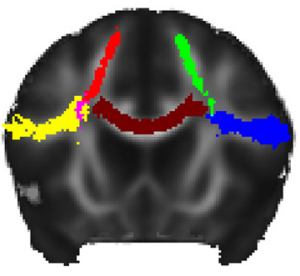

$y+18$
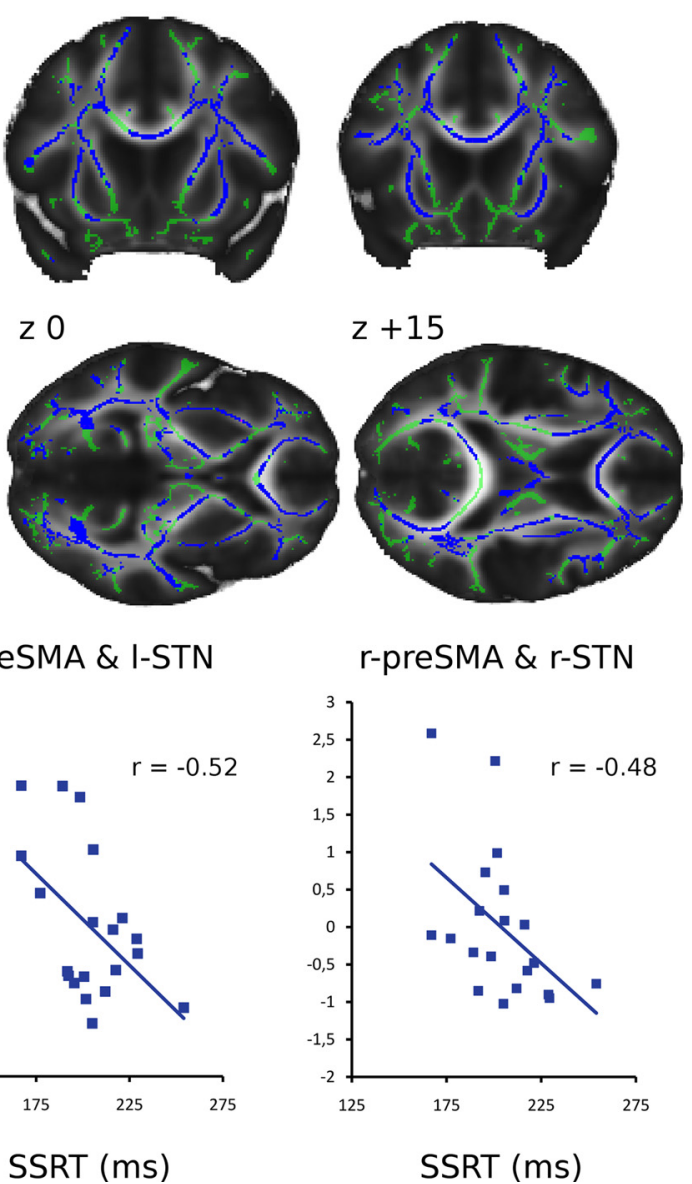

Figure 2. Probabilistic tractography and FA. Images are displayed using neurological convention. $\boldsymbol{A}$, The STN were manually delineated on filtered phase (shown) and susceptibility weighted images (not shown). Averaged across hemisphere, STN volume was $77 \pm 3.1 \mathrm{~mm}^{3}$ for Young and $66 \pm 3.1 \mathrm{~mm}^{3}$ for Old. The most likely MNI coordinates for I-STN and r-STN (Figure legend continues.) 
by calculating SSRT using the integration method (Verbruggen and Logan, 2009) averaged across $t 1$ and $t 2$, which gave almost identical results (linear positive correlation $r=0.975, R^{2}=0.95$ ). SSRT provides an index of stopping ability, such that individuals with faster SSRTs can be considered more efficient at stopping. For SSRT, a mixed ANOVA was performed with factors Group (Old, Young) and Stop condition (SA, SL-GR, SR-GL).

Stopping interference effects are a feature of selective conditions, with substantial delays evident for the hand still required to make a Go response, likely reflecting a global mechanism for rapid and transient braking of movement preparation, allowing resolution of pre-response conflict and response reprogramming, followed by execution of remaining response components (Coxon et al., 2007; Aron and Verbruggen, 2008). We quantified the stopping interference effect for the nonstopping hand (left hand for SR-GL, right hand for SL-GR) by calculating the response time delay relative to the mean response time of the same hand on Go trials. A mixed ANOVA was performed with factors Group (Old, Young) and Condition (SL-GR, SR-GL). The stopping interference effect was determined from trials in which the task was performed successfully.

Image acquisition and processing. A Siemens 3 T Magnetom Trio MRI scanner with standard head coil was used for image acquisition. For all subjects, the following structural scans were acquired within 1 week of behavioral testing: (1) a T1-weighted magnetization prepared rapid gradient echo image; repetition time (TR), $2300 \mathrm{~ms}$; echo time (TE), 2.98 $\mathrm{ms}$; flip angle, $9^{\circ}$; matrix, $240 \times 256$; 160 sagittal slices; $1.1 \times 1 \times 1 \mathrm{~mm}$ voxel resolution; (2) a $\mathrm{T} 2{ }^{*}$-weighted image for susceptibility weighted imaging using a fast low-angle shot sequence (FLASH); TR, $30 \mathrm{~ms}$; TE, 20 ms; flip angle, $12^{\circ}$; matrix, $192 \times 256$; 104 axial slices; $0.9 \times 0.9 \times 1.2 \mathrm{~mm}$ voxel resolution; and (3) a diffusion weighted single-shot spin-echo echoplanar image for diffusion tensor imaging (DTI); TR, $7200 \mathrm{~ms}$; TE, $81 \mathrm{~ms}$; flip angle, $90^{\circ}$; matrix, $96 \times 96$; 56 sagittal slices of thickness 2.2 $\mathrm{mm}$ with $0.66 \mathrm{~mm}$ gap; $2.19 \times 2.19 \times 2.86 \mathrm{~mm}$ voxel resolution. Diffusion sensitizing gradients were applied at a $b$ value of $1000 \mathrm{~s} / \mathrm{mm}^{2}$, along 64 non-collinear directions. An image with no diffusion weighting (b0) was also acquired.

Susceptibility weighted imaging processing. To generate the susceptibility weighted image, the magnitude and phase components of the FLASH acquisition were processed using SPIN (Magnetic Resonance Imaging Institute for Biomedical Research, Detroit, MI). The phase image was high-pass filtered in the spatial domain $(64 \times 64$ voxels $)$ to remove low-frequency spatial components, and a positive phase mask was applied. Subsequently, the magnitude image was multiplied by the processed phase image, resulting in a susceptibility weighted image with enhanced contrast in areas of high iron content (Haacke et al., 2009).

Diffusion weighted image processing. Diffusion data were analyzed using the FMRIB (Functional MRI of the Brain) Software Library, FSL (Oxford University, Oxford, UK). The $b 0$ image of each subject was skull-stripped using the brain extraction tool, data were corrected for subject motion and eddy-current induced geometrical distortions, and the diffusion sensitizing gradients ("bvecs") were rotated to correct for motion. Using the diffusion toolbox, the diffusion tensor model was fit to the data, from which fractional anisotropy (FA) images were calculated.

$\leftarrow$

(Figure legend continued.) were $-9,-12,-5$ and $10,-11,-5$, respectively. Cortical masks were defined in MNI space and transformed to subject DTI space. The 3 D rendering shows probabilistic tracts ( $>95 \%$ of participants) from r-IFC to r-preSMA and r-STN (dark and light blue, respectively) and from preSMA to STN in the left (red) and right (green) hemisphere. $\boldsymbol{B}$, ROIs from tract multiplication procedure (top row), coronal slice profile, and voxelwise TBSS analysis of FA (bottom row). ROIs are color coded as follows: r-IFC, blue; r-preSMA, green; r-STN, light blue; CC, brown; I-IFC, yellow; I-preSMA, red; I-STN, pink. Voxels with significantly reduced FA in the old group are shown in blue (TFCE correction) and are overlaid on the TBSS skeleton (green). Coronal slices with significantly reduced FA in the elderly are marked by $\times$ (after Bonferroni's correction). Note that almost all slices anterior to the anterior commissure (AC) are significant. C, Correlations with mean FA across all participants (left) and for tract connection strength in the old group (right).
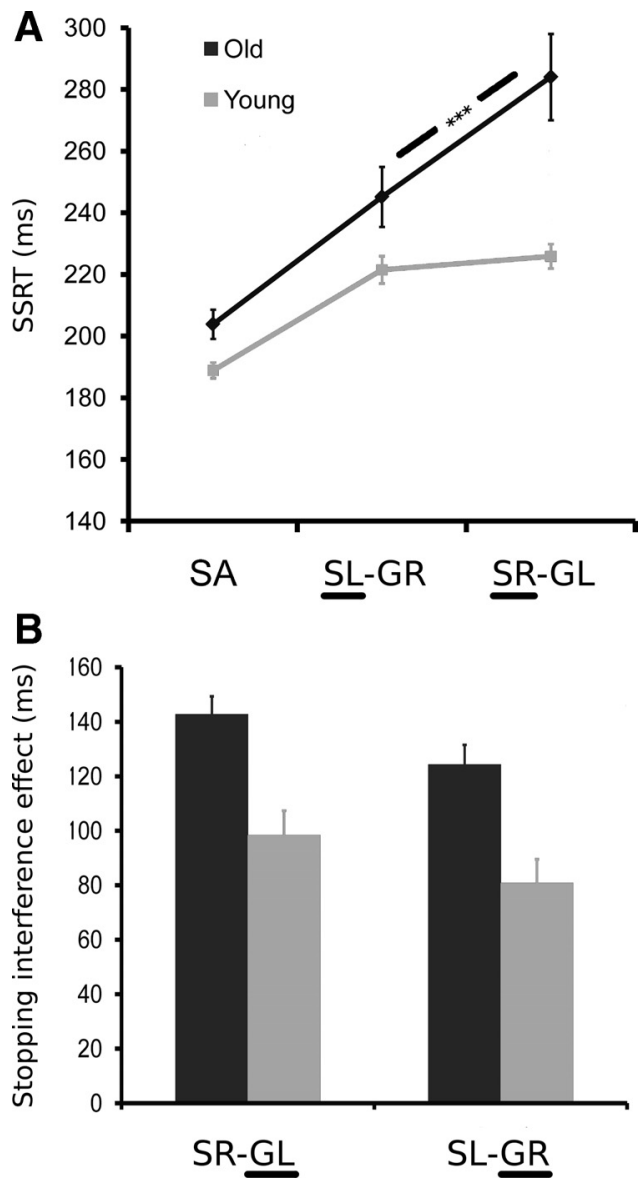

Figure 3. Stop task performance. $A$, SSRT is shown for each stop condition (SA, SL-GR, SR-GL) and group. For all conditions, SSRT was significantly prolonged for the old group (see Results). $B$, Stopping interference effect (response time delay relative to Go trials) is shown for each selective condition (Go Left during SR-GL, Go Right during SL-GR) and group. Error bars represent SES.

Voxelwise analysis of FA using tract-based spatial statistics. The FA images were used as input for tract-based spatial statistics (TBSS) (Smith et al., 2006). This involved registering all subjects' FA images to a common space (the FA158 MNI space template) using a combination of affine and nonlinear registration, creating the mean FA image, eroding it to a skeleton, and thresholding the skeleton at FA $>0.25$. The resulting alignment-invariant representation of the central trajectory of white matter pathways was used for voxelwise statistical analysis (randomize, 10,000 permutations). The contrast Old $<$ Young was examined using threshold-free cluster enhancement (TFCE) (Smith and Nichols, 2009), with correction for multiple comparisons at $\alpha<0.05$. To test whether there was evidence of an anterior-posterior white matter gradient, coronal slice profiles were determined (Sullivan and Pfefferbaum, 2006). The mean FA of all skeleton voxels superior to the anterior commissureposterior commissure line was calculated for each coronal slice, and one-tailed $t$ tests were used to compare groups (with Bonferroni's correction for the number of slices).

Cortical seed masks. Cortical seed masks for probabilistic tractography were determined in MNI space and transformed to subject diffusion space using the inverse of the TBSS registrations. The r-IFC was determined by the union of Brodmann's areas 44 and 45 (probability for each area $>50 \%$ ). The preSMA was defined using the automated anatomical labeling template (Tzourio-Mazoyer et al., 2002), rostral to a vertical line through the anterior commissure (i.e., $y>0$ ) (Johansen-Berg et al., 2004).

Manual delineation of the STN. FSLview was used to display the filtered phase and susceptibility weighted images simultaneously. The STN was located as hyperintense on the phase image, lateral and anterior to the 
Table 1. Mean FA from ROIs determined using probabilistic tractography

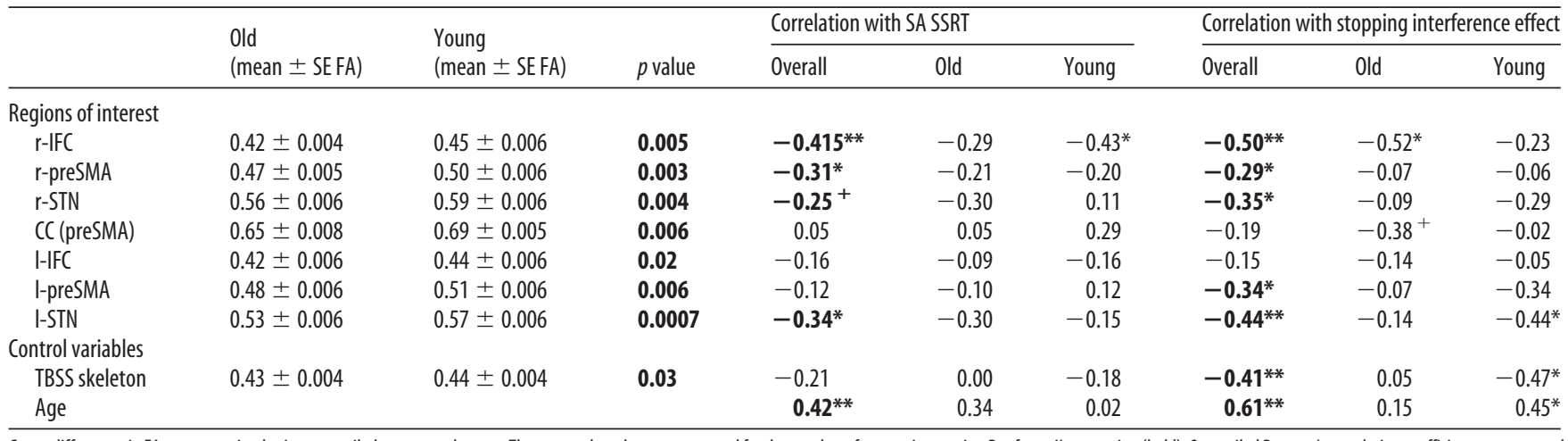

Group differences in FA were examined using one-tailed, two-sample $t$ test. The reported $p$ values are corrected for the number of comparisons using Bonferroni's correction (bold). One-tailed Pearson's correlation coefficients are reported for the relationship between FA and measures of stopping performance, controlling for mean skeleton FA (significant correlations across all participants shown in bold for clarity; ${ }^{*} p<0.05$; ${ }^{* *} p<0.01$; ${ }^{+} p<0.07$ ).

Table 2. Regression analysis predicting SSRT from connection strength of probabilistic tracts

\begin{tabular}{|c|c|c|c|c|c|c|c|c|c|c|c|c|}
\hline \multirow[b]{2}{*}{ Model } & \multicolumn{4}{|c|}{$\begin{array}{l}\text { All participants (Adjusted } R^{2}=0.315, F_{(8,30)}= \\
3.2, p=0.01 \text { ) }\end{array}$} & \multicolumn{4}{|c|}{ Old (Adjusted $R^{2}=0.40, F_{(7,12)}=2.82, p=0.05$ ) } & \multicolumn{4}{|c|}{$\begin{array}{l}\text { Young (Adjusted } R^{2}=-0.08, F_{(7,11)}= \\
0.8, p=0.60)\end{array}$} \\
\hline & B & SE & $\beta$ & $r$ & B & SE & $\beta$ & $r$ & B & SE & $\beta$ & $r$ \\
\hline Constant & 179.1 & 11.4 & & & 204.5 & 19.9 & & & 173.8 & 11.6 & & \\
\hline r-IFC and r-STN & -0.06 & 0.11 & -0.08 & -0.09 & -0.29 & 0.18 & -0.36 & -0.18 & 0.17 & 0.14 & 0.40 & 0.19 \\
\hline r-preSMA and r-STN & -0.13 & 0.07 & $-0.26^{+}$ & -0.21 & -0.18 & 0.09 & $-0.41^{+}$ & $-0.48^{*}$ & 0.21 & 0.17 & 0.39 & 0.08 \\
\hline r-preSMA and I-preSMA & 0.00 & 0.00 & 0.07 & -0.11 & 0.01 & 0.01 & 0.18 & -0.05 & 0.00 & 0.01 & 0.03 & 0.05 \\
\hline I-IFC and I-STN & 0.45 & 0.38 & 0.17 & 0.06 & 0.47 & 0.83 & 0.13 & 0.05 & 0.52 & 0.39 & 0.04 & 0.23 \\
\hline Age & 0.29 & 0.15 & $0.34^{*}$ & $0.42^{* *}$ & & & & & & & & \\
\hline
\end{tabular}

For all models and parameter estimates, the variance inflation factor ranged from 1.186 to 1.822 (i.e., evidence of multicolinearity was minimal). Significant contributions to model fit and zero-order Pearson's correlation coefficients are marked in bold (level of significance: ${ }^{*} p<0.05 ;{ }^{* *} p<0.01 ;{ }^{+} p<0.09$ ).

superior border of the red nucleus, and superior to the substantia nigra (Lucerna et al., 2002; Dormont et al., 2004). Three independent raters manually outlined the l-STN and r-STN of each participant. The STN masks were determined for each subject separately, by transforming to diffusion space the voxels labeled by at least two of three raters on the susceptibility weighted images. Whether calculated for all participants or each group separately, intraclass correlation coefficients for the two raters with the most similar STN volumes were $>0.875$ (for both 1-STN and $\mathrm{r}$-STN). To view the location of STN in MNI space, the T1 image of each subject was warped to match the MNI152 T1 image using a combination of affine and nonlinear registration. The registrations were then applied to the phase and STN images, and the transformed STN images were summed across subjects (Fig. 2A).

Probabilistic tractography. In subject diffusion space, a probabilistic diffusion model that accommodates crossing fibers was applied to calculate probability distributions on fiber direction at each voxel (Behrens et al., 2003, 2007). Probabilistic tractography was run from cortical seed masks, constrained by a target mask, to delineate the following tracts: (1) r-IFC to r-preSMA; (2) r-IFC to r-STN; (3) r-preSMA to r-STN; (4) r-preSMA to l-preSMA; (5) 1-preSMA to l-STN; (6) 1-IFC to l-preSMA; and (7) 1-IFC to l-STN. Tracts from 1-IFC were included as control tracts, because a large body of work emphasizes the role of r-IFC in inhibitory control. Starting from each voxel in the seed region, 10,000 samples were generated with a curvature threshold of 0.2 , and the samples reaching the target mask were retained. For within-hemisphere tractography, a mask was used to exclude tracts crossing the midline. For between-hemisphere tractography, a mask was used to only include tracts passing through the corpus callosum (CC). The total number of samples reaching the target was divided by the number of voxels in the seed mask, and resulting values were used as an indicator of relative connection strength across participants (for a similar approach, see Forstmann et al., 2010). The connection strengths represent the extent to which the dominant paths through the diffusion field (a reflection of white matter tract orientations) passes from seed to target for each individual. Tracts were entered, along with age, as independent variables for multiple linear regression analysis (SPSS version 16; IBM) predicting (1) SSRT and (2) the stopping interference effect (average of SL-GR and SR-GL). The model was fit initially for all participants and then for the old and young groups separately.

Mean FA region of interest analysis. To determine the probable spatial trajectory of each tract, the maps were thresholded at a low level to remove noise (this was $0.02 \%$ of the maximum value in the connectivity map) (Aron et al., 2007), transformed to MNI space (using the TBSS registrations), binarized, and summed across participants. Voxels that were present in $>95 \%$ of participants' maps were retained (Fig. $2 \mathrm{~A}, 3 \mathrm{D}$ render). Given near-perfect overlap in the vicinity of a seed/target node, a multiplication procedure was used to determine voxels common to white matter tracts to/from each region [to avoid non-independent tract regions of interest (ROIs)]. For simplicity, the ROIs are labeled according to the common seed/target node (e.g., the r-IFC ROI was determined by the multiplication of the tract between r-IFC and r-preSMA and the tract between r-IFC and r-STN). The resulting MNI space tract ROIs (Fig. $2 \mathrm{~B}$ ) were used to extract the mean FA from each subjects' TBSS skeleton image. Thus, the value for each ROI can be thought to reflect the integrity of white matter projections to/from the other nodes (e.g., r-IFC contains voxels projecting to/from both r-preSMA and r-STN). Mean FA was correlated with (1) SSRT and (2) the stopping interference effect, and significant correlations were subjected to partial correlation analysis controlling for mean skeleton FA and age (SPSS version 16; IBM).

The analysis approach outlined above capitalizes on previous work, which has identified r-IFC, preSMA, and STN as critical nodes in a network for stopping and/or braking action (see Introduction). We therefore used an ROI approach given our strong a priori hypothesis of where we expected to observe effects. Below we report results for our two primary dependent measures, mean tract FA and tract strength, and then complement these findings with a whole-brain TBSS approach that lends support to the specificity of the results of the ROI-based analyses. 
A
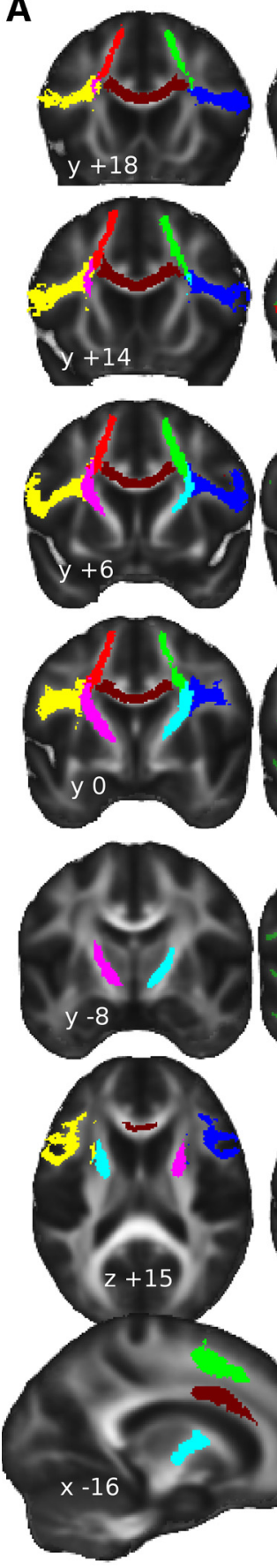
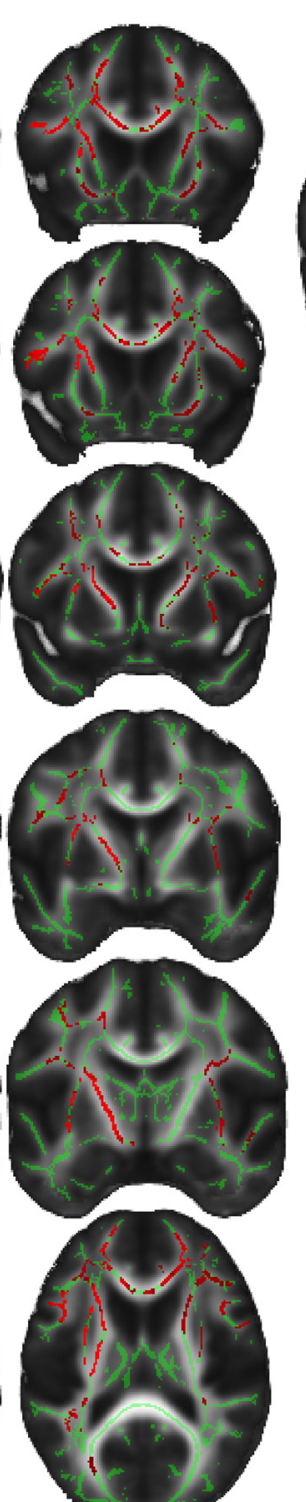

B Overall (MNI $49317, \mathrm{t}=4.12$, partial $r=-0.59$ )

Controlling for age $\&$ mean skeleton FA

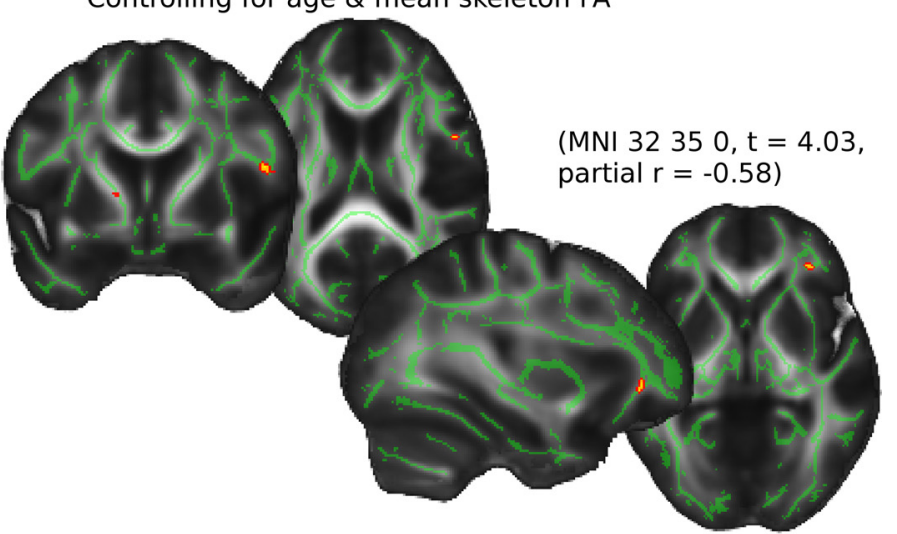

Overall (MNI-12 $15, \mathrm{t}=4.41$, partial $r=-0.59$ )

Controlling for age $\&$ mean skeleton FA or Go variability

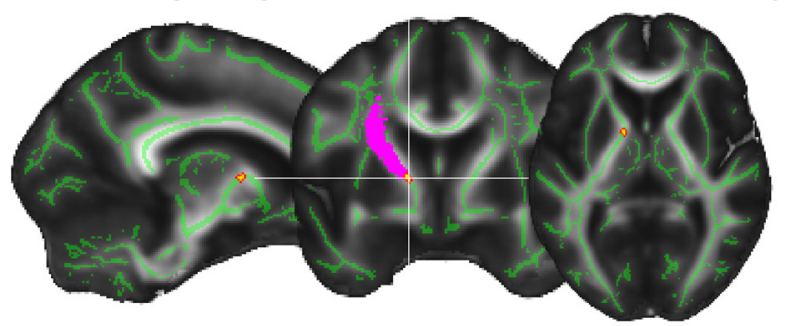

Old group (MNI -20 $1212, t=5.41$, partial $r=-0.69$ ) Controlling for age $\&$ mean skeleton FA

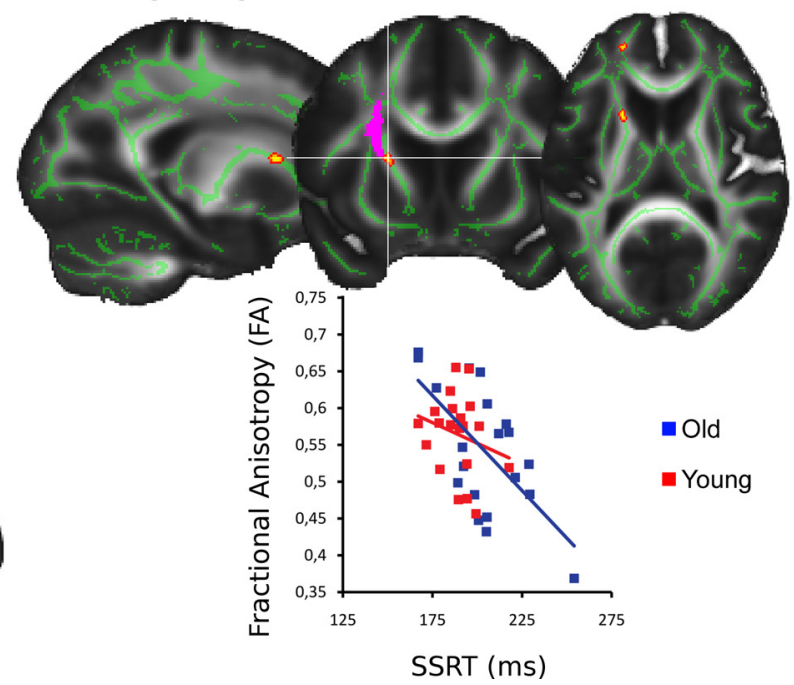

Figure 4. TBSS regression analyses predicting SA SSRT. A, Significant voxels in which lower FA is associated with prolonged SA SSRT across all participants (correction for multiple comparisons using TFCE, $\alpha<0.05$ ). Tract ROls are displayed for comparison (color coding same as Fig. 2). B, Significant clusters when additionally controlling for age and mean skeleton FA or Go variability across all participants (top) and in the old group separately (bottom). Note the cluster falling within the I-STN tract mask. Significant clusters are dilated by one voxel for improved visualization.

\section{Results}

\section{Movement prevention experiment}

SSRT

SSRT is shown in Figure $3 A$. There were main effects of Group $\left(F_{(1,38)}=15.3, p<0.001\right)$ and Stop condition $\left(F_{(2,76)}=46.3, p<\right.$ $0.001)$. The interaction of Group $\times$ Stop condition was also significant $\left(F_{(2,76)}=6.9, p=0.001\right)$. For all conditions, older adults needed more time to inhibit their response (SA: Old, $204 \pm 5 \mathrm{~ms}$; Young, $188 \pm 3 \mathrm{~ms} ; p<0.01$; SL-GR: Old, $245 \pm 10 \mathrm{~ms}$; Young, $221 \pm 4 \mathrm{~ms}$; $p=0.02$; SR-GL: Old, $284 \pm 14$ ms; Young, $226 \pm 4$ ms; $p<0.001$; after Bonferroni's correction). The group difference was largest for the SR-GL condition. This may reflect a problem initiating a unimanual response with the nondominant hand because, in contrast to the young group, there was a significant difference between SL-GR and SR-GL for the old group $(p<0.001)$. Consistent with this view, studies show increased transcallosal motor overflow in older adults during unimanual movements (Addamo et al., 2007; Talelli et al., 2008; Giovannelli et al., 2009). However, the effect of hand dominance cannot be known for certain without testing both left- and right-handed individuals.

Overall, the correlation between nonselective (SA SSRT) and selective (SL-GR and SR-GL SSRT) stopping was significant ( $r=$ 
0.61 , one-tailed $p<0.001)$ and remained significant after controlling for age $(r=0.50$, one-tailed $p=0.001)$. Because selective SSRTs may also be influenced by the additional sensory discrimination and motor requirements over and above stopping per se, we concentrated on the "purest" estimate of the stopping process, SA SSRT, when investigating relationships with stopping network integrity.

\section{Stopping interference effect (Go response time delay)}

As expected, substantial stopping interference effects were observed (Fig. $3 B$ ). The interference effect was greater in the older adults (main effect of Group, $F_{(1,38)}=16.8, p<0.001$ ) and for the selective condition requiring a response by the dominant hand (i.e., SR-GL) (main effect of Condition, $F_{(1,38)}=24.7, p<0.001$ ). The Group $\times$ Condition interaction was not significant.

\section{Go task performance}

On average, the old group responded slightly later (Old, $27 \pm 3$ $\mathrm{ms}$; Young, $17 \pm 2 \mathrm{~ms} ; p<0.05)$ and were slightly more variable (Old, $50 \pm 2 \mathrm{~ms}$; Young, $34 \pm 2 \mathrm{~ms} ; p<0.001$ ) than the young group. Although statistically significant, these age-related differences were substantially less than previous stop-signal studies using choice reaction time for the Go task (Williams et al., 1999; Bedard et al., 2002). This suggests that the present task provided relatively strong control over the timing of the Go process, increasing confidence in the interpretation of group differences in the Stop process. Importantly, the calculations of both SSRT and the stopping interference effect control for individual differences in Go task performance.

\section{Diffusion imaging}

Age-related change in FA

Widespread age-related FA decline was observed throughout the TBSS skeleton, with more pronounced effects in anterior brain regions (Fig. $2 \mathrm{~B}$ ). The mean FA values extracted from each ROI are reported in Table 1. For all ROIs investigated, mean FA was significantly reduced in the old group.

\section{Correlations between FA and behavior}

The main question addressed in the present study was whether task-specific frontal white matter (r-IFC and bilateral preSMASTN) is predictive of behavioral inhibition. We tested for brainbehavior relationships in tractography defined ROIs in each hemisphere, accounting for any general relationship with wholebrain FA.

Lower mean FA was associated with longer SSRT (i.e., worse stopping; Fig. 2C, left graph). Across all participants, this relationship was significant for the r-IFC, r-preSMA, r-STN, and l-STN white matter ROIs. These regions remained significant after controlling for the relationship between SSRT and global FA (partial correlations controlling for mean skeleton FA; Table 1). For the r-IFC ROI, FA remained a significant predictor of SSRT after additionally controlling for age $(r=-0.33, p<0.05)$.

There were no significant relationships between FA and selective SSRT (average of SL-GR and SR-GL) within the stopping network ROIs, for either all participants or each group separately. Selective SSRT is likely influenced by extra sensory discrimination and motor requirements, and thus relationships with white matter integrity within the identified network may be masked by these additional sources of individual differences, which depend on processing in other brain regions.

If global stopping contributes to the interference effect or if the interference effect reflects transient response "braking" to
Table 3. Whole-brain TBSS analysis testing for a negative relationship between FA and SA SSRT

\begin{tabular}{llrrrrr}
\hline Location & Voxels $x$ & $y$ & $z$ & $t$ & Partial $r$ \\
\hline All participants, SA SSRT and FA, controlling & & & & & & \\
$\quad$ for age and mean skeleton FA & & & & & & \\
$\quad$ L Forceps major & 31 & -25 & -72 & 14 & 4.32 & -0.61 \\
L Crus II & 21 & 12 & -69 & -35 & 4.64 & -0.63 \\
L Forceps major & 17 & -14 & -43 & 12 & 4.74 & -0.64 \\
R IFC anterior/R IFOF & 14 & 32 & 35 & 0 & 4.03 & -0.58 \\
R IFC tract mask & 11 & 49 & 3 & 17 & 4.12 & -0.59 \\
I-STN tract mask & 11 & -12 & 1 & 5 & 4.18 & -0.59 \\
All participants, SA SSRT and FA, controlling & & & & & & \\
$\quad$ for age and Go variability & & & & & & \\
L STN tract mask & 10 & -12 & 1 & 5 & 4.41 & -0.61 \\
L White matter near V5 & 10 & -48 & -56 & -5 & 4.51 & -0.62 \\
L Crus II & 10 & 12 & -69 & -35 & 3.8 & -0.56 \\
Old group, SA SSRT and FA, controlling for & & & & & & \\
$\quad$ age and mean skeleton FA & & & & & & \\
L STN tract mask & 23 & -20 & 12 & 12 & 5.41 & -0.69 \\
CC genu & 23 & -3 & 29 & 2 & 4.94 & -0.66 \\
R White matter near central sulcus & 19 & 48 & -16 & 49 & 4.2 & -0.60 \\
L Forceps major & 17 & -26 & -68 & 14 & 5.3 & -0.68 \\
L STN tract mask & 17 & -15 & 5 & 8 & 3.96 & -0.57 \\
L Forceps minor & 13 & -20 & 47 & 12 & 4.46 & -0.62 \\
\hline
\end{tabular}

Clusters surviving a threshold of $p<0.001$ one-tailed, and consisting of at least 10 contiguous voxels are reported $L$, Left; R, right; R IFOF, inferior fronto-occipital fasciculus.

buy time for action reprogramming (Frank, 2006; Isoda and Hikosaka, 2008; Neubert et al., 2010), similar behavior-structure correlations should be observed, particularly for the STN. This was indeed the case because lower mean FA was associated with a greater stopping interference effect (Fig. 2C). After controlling for the relationship with global FA, the r-IFC, along with bilateral preSMA and STN ROIs were significant (Table 1). The r-IFC and l-STN ROIs remained a significant predictor of the interference effect after additionally controlling for age $(r=-0.40, p<0.01$; and $r=-0.29, p<0.05$, respectively).

\section{Relationship between tract strength and behavior}

Although FA is a useful and widely reported metric of white matter integrity, it is also sensitive to the underlying fiber complexity (e.g., in regions where white matter tracts cross each other). Because probabilistic tractography was performed using a dual-fiber model, we used the results as an indication of relative tract strength across individuals.

A multiple regression model indicated that tract connection strength is a significant predictor of SSRT when all participants are included (adjusted $R^{2}=0.315, p=0.01$ ) or when the model is calculated for the older adults only (adjusted $R^{2}=0.40, p=$ $0.05)$. For the model including all participants (Table 2 ), a significant negative regression coefficient was observed for the tract seeded in 1-preSMA targeting STN $(t=-2.1, p<0.05)$ along with marginal effects for the tract between $r$-preSMA and STN $(t=-1.7, p=0.09)$ and for age $(t=1.9, p=0.06)$. In the old group, the negative relationship between tracts seeded in preSMA targeting STN and SSRT was striking, such that lower tract strength was associated with longer SSRT (left, $t=-2.5, p<$ 0.05 ; right, $t=-2.0, p=0.06$; Fig. $2 C$, right two graphs; Table 2 ). This relationship remained even after a partial correlation analysis controlling for potentially confounding effects of age and STN target mask size (1-preSMA and 1-STN, $r=-0.55, p=0.01$; r-preSMA and r-STN, $r=-0.48, p<0.05)$. For the young group, there was no significant correlation (1-preSMA and 1-STN, $r=$ $-0.12, p=0.32$; r-preSMA and r-STN, $r=0.07, p=0.39$ ). 
Furthermore, the strength of the preSMASTN relationship in the older adults differed significantly with respect to young adults as shown by a significant difference in slope and correlation coefficient (one-tailed $t$ test: l-preSMA and 1-STN, $p<0.05$ and $p=0.078$, respectively; r-preSMA and r-STN, both $p<$ 0.05).

To test the specificity of the preSMASTN result, we performed a control tract analysis. SMA-proper is known to also have direct projections to STN (Inase et al., 1999; Nambu et al., 2002), but this region is not considered critical for the stopping process. We performed tractography between SMA-proper and STN in each hemisphere to see whether the strength of this adjacent tract predicted SSRT. No significant relationship was observed, for either all participants (1-SMA-proper and l-STN, $r=-0.07, p=0.32$; r-SMAproper and r-STN, $r=0.07, p=0.32$ ) or the Old group separately (1-SMA-proper and l-STN, $r=-0.08, p=0.38$; r-SMAproper and r-STN, $r=-0.16, p=0.24$ ).

Running the regression models with the stopping interference effect or Go response time variability as the dependent variable revealed age as the only significant regression coefficient (all participants: age $t=3.2, p=0.003$; and age $t=4.3, p<0.001$, respectively). Regression models performed on each group separately were not significant (both $p>$ 0.3 ). That we observe a preSMA-STN relationship for SSRT but not Go response time variability is important because this indicates specificity to the Stop as opposed to the Go task process.

Whole-brain TBSS regression analyses We supplement our hypothesis-driven approach with analogous whole-brain TBSS skeleton regression analyses to search for converging evidence. First, SA SSRT, demeaned across the entire sample, was regressed against FA using randomize $(10,000$ permutations). The results of this analysis (Fig. 4A) show a frontal predominance with lower FA associated with longer SA SSRT (significant voxels survived correction for multiple comparisons using TFCE, $\alpha<0.05)$. In general, a strong accordance was observed with the overall correlations reported in the ROI analysis, with significant voxels clearly overlapping with the tractography-derived network.

In subsequent analyses, we thresholded the statistical map resulting from randomize permutation testing at $p<0.001$, onetailed, with a cluster extent threshold of $\geq 10$ contiguous skeleton voxels. This approach has been taken in several published studies (Boorman et al., 2007; Mars et al., 2009; Neubert et al., 2010) and is considered reasonably stringent given that the number of voxels in the TBSS skeleton is of an order of magnitude lower than FMRI datasets. improved visualization.
A

\section{Controlling for age \& skeleton FA or Go variability}

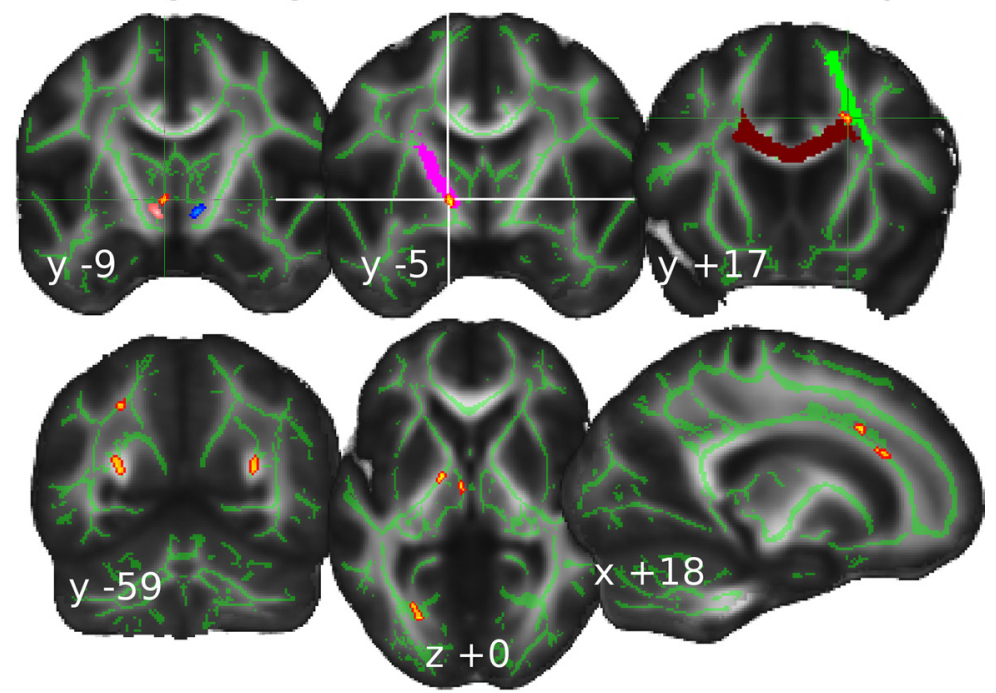

B
Old group ( $\mathrm{MNI}-13-50, t=4.93$, partial $r=-0.66$ ) Controlling for age \& mean skeleton FA
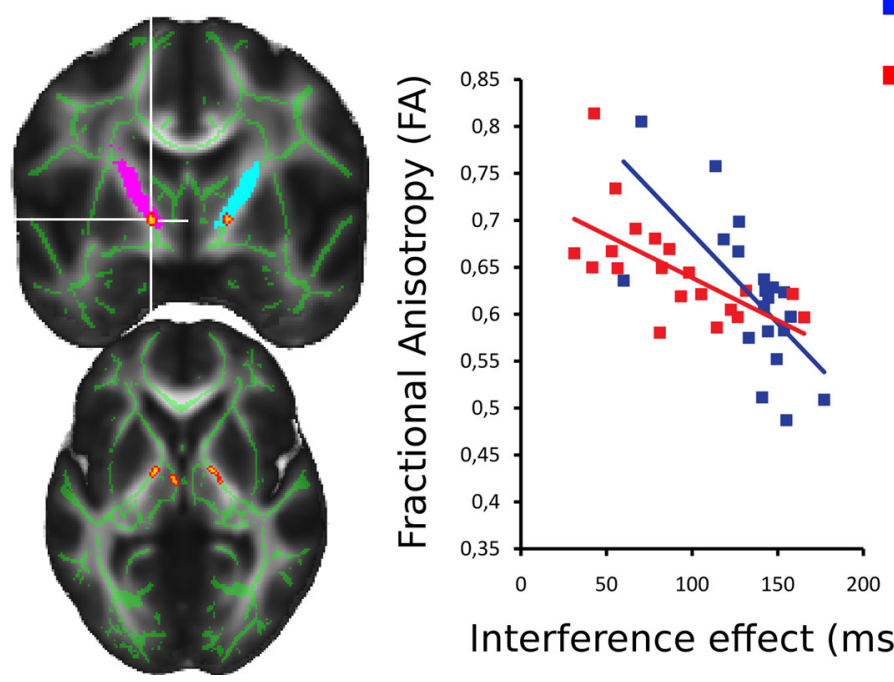

Interference effect (ms)

Figure 5. TBSS regression analyses predicting stopping interference effects. Significant clusters in which lower FA is associated with increased stopping interference effects, controlling for age and mean skeleton FA or Go variability: $\boldsymbol{A}$, across all participants; $\boldsymbol{B}$, in the old group separately. Note the cluster falling within the I-STN tract mask. Significant clusters are dilated by one voxel for

We examined whether reduced FA predicted prolonged SSRT, controlling for age and mean skeleton FA (Fig. 4 B; Table 3). Two clusters in the vicinity of the r-IFC tract ROI were observed, along with a cluster within the l-STN tract ROI. This latter 1-STN cluster also survived an analysis controlling for variability in Go task performance (MNI coordinates, $-12,1,5 ; t=4.41$, partial $r=$ -0.61). This indicates that the relationship between FA and SSRT observed in a location spatially compatible with 1-STN projections cannot be explained by individual differences in Go task performance. When looking at the old group separately, the strongest effect we observed also fell within the l-STN tract ROI (Fig. $4 B$, bottom; Table 3 ), complementing the results of the tract strength analysis. 
Table 4. Whole-brain TBSS analysis testing for a negative relationship between FA and the stopping interference effect

\begin{tabular}{|c|c|c|c|c|c|c|}
\hline Location & Voxels & $x$ & $y$ & $z$ & $t$ & Partial $r$ \\
\hline \multicolumn{7}{|c|}{$\begin{array}{l}\text { All participants, stopping interference and } \\
\text { FA, controlling for age and mean } \\
\text { skeleton FA }\end{array}$} \\
\hline R Forceps major & 46 & 28 & -55 & 15 & 3.97 & -0.54 \\
\hline L Forceps major & 41 & -26 & -57 & 15 & 4.54 & -0.63 \\
\hline $\mathrm{RCC}$ preSMA tract mask & 28 & 17 & 26 & 23 & 4.17 & -0.59 \\
\hline LSTN tract mask & 22 & -13 & -5 & 0 & 5.51 & -0.70 \\
\hline LSTN adjacent nucleus & 16 & -4 & -9 & 0 & 3.85 & -0.56 \\
\hline $\mathrm{R}$ Optic radiation & 15 & 27 & -73 & 21 & 4.46 & -0.62 \\
\hline L Optic radiation & -15 & -23 & -69 & 0 & 4.46 & -0.62 \\
\hline L Optic radiation & 13 & -37 & -41 & 6 & 4.18 & -0.59 \\
\hline $\mathrm{R}$ Optic radiation & 13 & 42 & -19 & -14 & 4.72 & -0.64 \\
\hline L White matter near anterior IPS & 12 & -25 & -56 & 39 & 3.93 & -0.57 \\
\hline L Corticospinal tract & 11 & -21 & -29 & 41 & 3.75 & -0.55 \\
\hline RCC preSMA tract mask & 11 & 18 & 17 & 33 & 4.48 & -0.62 \\
\hline L Optic radiation & 11 & -42 & -33 & -7 & 3.58 & -0.54 \\
\hline \multicolumn{7}{|c|}{$\begin{array}{l}\text { All participants, stopping interference and } \\
\qquad \text { FA, controlling for age and Go variability }\end{array}$} \\
\hline L Forceps major & 29 & -22 & -53 & 13 & 3.67 & -0.54 \\
\hline R Forceps major & 23 & 28 & -56 & 14 & 3.53 & -0.53 \\
\hline $\mathrm{RCC}$ preSMA tract mask & 22 & 18 & 27 & 23 & 4.01 & -0.58 \\
\hline L STN tract mask & 19 & -13 & -6 & 0 & 5.41 & -0.69 \\
\hline $\mathrm{R}$ Corticospinal tract & 13 & 24 & -17 & 49 & 4.32 & -0.61 \\
\hline \multicolumn{7}{|c|}{$\begin{array}{l}\text { Old group, stopping interference and FA, } \\
\text { controlling for age and mean skeleton FA }\end{array}$} \\
\hline L Forceps major & 55 & -26 & -57 & 15 & 4.53 & -0.62 \\
\hline $\mathrm{RCC}$ preSMA tract mask & 52 & 15 & 25 & 21 & 4.7 & -0.64 \\
\hline R Forceps major & 27 & 28 & -54 & 16 & 3.85 & -0.56 \\
\hline L STN vicinity of nucleus & 20 & -3 & -10 & 1 & 4.05 & -0.58 \\
\hline R IFC anterior/R IFOF & 17 & 42 & 33 & 4 & 4.74 & -0.64 \\
\hline R STN tract mask & 17 & 14 & -4 & 0 & 4.07 & -0.58 \\
\hline LSTN tract mask & 16 & -13 & -5 & 0 & 4.93 & -0.66 \\
\hline L IFC tract mask & 12 & -46 & 6 & 13 & 3.89 & -0.57 \\
\hline R Optic radiation & 12 & 28 & -18 & -7 & 5.95 & -0.72 \\
\hline L Optic radiation & 12 & -29 & -18 & -8 & 4.08 & -0.58 \\
\hline
\end{tabular}

Clusters surviving a threshold of $p<0.001$ one-tailed. At least 10 contiguous voxels are included. $L$, Left; $R$, right; $R$ IFOF, right inferior fronto-occipital fasciculus.

The same analyses predicting increased stopping interference effects revealed FA clusters within the l-STN tract mask, adjacent to the nucleus itself, and in the CC preSMA tract mask, while controlling for age and mean skeleton FA (Fig. 5A; Table 4). In addition, several clusters were observed in white matter connecting with visual cortical areas (forceps major and optic radiations bilaterally). Most of the relationships in posterior white matter disappeared when controlling for Go task variability, suggesting that these posterior observations may not be specific to the process of interest. In contrast, the relationships observed in the 1-STN (MNI coordinates, $-13,-5,0 ; t=5.41$, partial $r=-0.69$ ) and CC preSMA tract masks (MNI coordinates, 18, 27, 23; $t=$ 4.01, partial $r=-0.58$ ) remained when controlling for the Go task performance variability of each individual. Finally, examining the old group separately revealed clusters within the STN tract masks bilaterally (Fig. 5B), the l-IFC tract mask, and the CC preSMA tract mask (Table 4).

In summary, the whole-brain TBSS approach provides complementary evidence that the integrity of white matter pathways connecting STN are predictive of both outright stopping of actions and the interference observed when selectivity is required.

\section{Discussion}

In the present study, we investigated the effect of age on brainbehavior relationships within an anatomical model of cogni- tive action control consisting of r-IFC, preSMA, and STN. Individual differences in white matter integrity (FA) were shown to predict inhibitory control efficiency. Bilateral preSMA-STN tract strength significantly predicted stopping ability (SSRT), and this relationship was strongest in older adults as shown by a significant group difference in correlation coefficient and regression slope. Additional whole-brain analyses identified clusters within the STN tract ROIs in which FA predicted Stop task behavior even after controlling for Go task performance variability. Our data link age-related decline in inhibitory control with structural decline of STN projections. In doing so, we provide a neural correlate for the inhibition deficit theory of age-related cognitive decline. The strength of preSMA-STN tracts may be a useful biomarker of age-related declines in cognitive action control and, more generally, inhibitory function.

\section{Age-dependent and age-independent relationships between FA and stopping in task-relevant white matter}

The voxelwise analysis of FA confirmed decline of white matter integrity throughout the aging brain (see also Barrick et al., 2010). Such widespread reductions may contribute to the behavioral effects observed, because overall white matter is predictive of information processing speed in old age (Penke et al., 2010). However, we controlled for such a general relationship by partialling out mean skeleton FA.

Age-related degeneration was most pronounced in anterior fiber systems, signifying an anterior-posterior gradient of decline (Sullivan and Pfefferbaum, 2006). Importantly, right but not l-IFC white matter was correlated with stop task performance, indicating anatomically specific brain-behavior relationships over and above age and general white matter decline (Fig. 2C). A critical role of r-IFC in inhibitory control has been inferred previously from patients with frontal lesions (Aron et al., 2003), but there is ongoing debate as to whether r-IFC detects the stop signal, triggers the stopping process, or both (Levy and Wagner, 2011). Our results in the healthy human brain, along with other recent findings (Madsen et al., 2010; King et al., 2012), point toward the importance of white matter underlying r-IFC for stopping.

Across all participants, we found that SSRT was predicted by FA in r-IFC, and preSMA and STN ROIs bilaterally (Table 1). The overall correlations for r-IFC and r-preSMA appeared to be driven by both age groups, whereas those for l-STN and r-STN were more driven by the old group. Results complementary to ours have been reported in children 7-13 years old in which better response inhibition was associated with FA underlying r-IFC and r-preSMA (Madsen et al., 2010), but they did not examine tracts connecting with STN. In the present study, we provide clear evidence that the integrity of white matter connecting anatomically defined STN predicts stopping performance. We confirm this with whole-brain TBSS regression analyses and show that the result cannot be explained by variability in Go task performance (Fig. 4). That is, the observed relationship is specific to the Stop process of this task. We also provide the first evidence that the integrity of white matter projecting to l-STN predicts stopping.

\section{Tract strength analysis reveals a critical role of preSMA-STN connections in stopping}

It is unclear whether r-IFC influences STN directly or via the preSMA. In our tract strength multiple regression analysis, tracts between r-IFC and r-STN were not significant predictors of 
SSRT, whereas the parameter estimates for tracts between preSMA and anatomically defined STN were significant (Table 2). Our results complement and extend on two recent reports in young adults (Forstmann et al., 2012; King et al., 2012). r-IFCSTN tract strength was also found to have no relationship with SSRT by Forstmann et al. (2012). Interestingly, they failed to find a relationship with preSMA-STN tract strength, instead reporting that the strength of the tract between anterior cingulate cortex (ACC) and STN predicts stopping ability. The reason for this discrepancy is unclear. Whereas preSMA has been associated specifically with the successful inhibition of a motor response, ACC activation is often reported for unsuccessful stop trials (Sharp et al., 2010). A tract strength analysis was not reported in the study by King et al. (2012), nor did they treat preSMA and SMA-proper as separate cortical regions despite major differences in anatomical connectivity (Johansen-Berg et al., 2004). We extend on these studies by showing that preSMA, but not SMA-proper connections with STN, predict stopping efficiency.

There is no convincing account of how stopping-related activity in the right hemisphere exerts an influence over the left hemisphere, which is known to be dominant for movement preparation. Evidence that $\mathrm{r}-\mathrm{STN}$ and $\mathrm{l}-\mathrm{STN}$ are directly connected is scarce, with one study reporting time-locked contralateral action potentials at a likelihood of only 3\% (Walker et al., 2011). Granger causality analysis on fMRI stop-task data argues against a direct influence of r-IFC on STN (Duann et al., 2009). Pairedpulse transcranial magnetic stimulation of r-IFC and left motor cortex during task-switch inhibition shows evidence of physiological inhibition, likely mediated by preSMA-STN interactions (Neubert et al., 2010). In our study, the relationship between tract strength and stopping was strongest between preSMA and l-STN. This tract may support stopping of movements initiated in the left hemisphere. Involvement of 1-STN in stopping is supported by the observation that unilateral deep brain stimulation of 1-STN impairs SSRT in Parkinson's disease patients performing similarly to controls (Ray et al., 2009). Based on our results, we tentatively propose that r-IFC interacts with STN via preSMA projections in each hemisphere.

However, we note that diffusion-based imaging measures reflect the strength of a structural connection and are not representative of physiological connectivity (i.e., the transmission of action potentials). Our results indicate that structural connection strength becomes a sensitive measure when integrity declines with aging, such that variability in connectivity influences behavior. The association between preSMA-STN tract connection strength and stopping ability in older adults is interesting in light of an fMRI study showing reduced STN activation during task switching with an inhibition component (Coxon et al., 2010). Reduced integrity of the pathway between preSMA and STN may lead to "structural disconnection" (O’Sullivan et al., 2001) and an inability to functionally modulate STN in older age.

\section{Integrity of tracts projecting to STN also predict individual differences in the stopping interference effect}

Interference manifests as delayed responding during selective stop trials and is likely the result of transient braking of all initiated movement and subsequent pre-response conflict resolution and action reprogramming (Frank, 2006; Coxon et al., 2007). The delays we observe are substantial, indicating that, to achieve a selective behavioral outcome in our paradigm, a nonselective neural inhibition mechanism is triggered. Functional imaging studies show that activity in the cognitive control network is present for outright stopping (Aron and Poldrack, 2006), task switch- ing (Coxon et al., 2010; Kenner et al., 2010), conflict-induced slowing in a conditional stopping paradigm (Aron et al., 2007), and incongruent stimulus-response mappings (Forstmann et al., 2008). Activity in preSMA is stronger for behaviorally selective compared with nonselective stop trials (Coxon et al., 2009) and r-IFC, preSMA, and STN activation scales with the magnitude of slowing on conditional stop trials (Aron et al., 2007). Our results are complementary by showing that, across all participants, greater stopping interference effects are associated with lower FA in the white matter connecting r-IFC, preSMA, and STN (Table 1).

Stopping interference effects were greater in the older adults. Our TBSS regression analyses (Table 4) revealed a cluster for the old group in the inferior fronto-occiptal fasciculus anterior to r-IFC, a tract previously related to conflict-induced slowing in young adults (Forstmann et al., 2008). We also saw correlations in posterior white matter (forceps major, optic radiations) connecting with visual cortex. This suggests that visual input specifying the stimulus-response mapping may contribute to some of the age-related increase in interference effects. However, forceps major associations appear task nonspecific because they were also observed when the same older adults participated in a balance control experiment (Van Impe et al., 2011). Here we establish that declining STN white matter integrity contributes to the age-related increase in the interference effect (Fig. 5). In addition, CC white matter (connecting 1-preSMA and r-preSMA) showed a relationship (Tables 1,4 ). These data suggest that increased interference effects in older adults may be a consequence of structural decline in the circuitry underlying both inhibitory control and interhemispheric communication required on selective trials.

In summary, we provide a structural basis for age-related declines in inhibitory control. We show that integrity of white matter within the STN tract ROI predicts both outright stopping (SSRT) and transient braking of response initiation to buy time for action reprogramming (stopping interference effects). Whereas tract connection strength between preSMA and striatum supports the modulation of response thresholds (Forstmann et al., 2010, 2011), our multiple regression analysis points to preSMA-STN connections in each hemisphere as important for expeditious stopping. The preSMA-STN tract manifests as a result of white matter decline, indicating that it may be a biomarker for age-related inhibitory deficits.

\section{References}

Addamo PK, Farrow M, Hoy KE, Bradshaw JL, Georgiou-Karistianis N (2007) The effects of age and attention on motor overflow production-a review. Brain Res Rev 54:189-204

Aron AR (2007) The neural basis of inhibition in cognitive control. Neuroscientist 13:214-228.

Aron AR, Poldrack RA (2006) Cortical and subcortical contributions to Stop signal response inhibition: role of the subthalamic nucleus. J Neurosci 26:2424-2433.

Aron AR, Verbruggen F (2008) Stop the presses: dissociating a selective from a global mechanism for stopping. Psychol Sci 19:1146-1153.

Aron AR, Fletcher PC, Bullmore ET, Sahakian BJ, Robbins TW (2003) Stopsignal inhibition disrupted by damage to right inferior frontal gyrus in humans. Nat Neurosci 6:115-116.

Aron AR, Behrens TE, Smith S, Frank MJ, Poldrack RA (2007) Triangulating a cognitive control network using diffusion-weighted magnetic resonance imaging (MRI) and functional MRI. J Neurosci 27:3743-3752.

Bäckman L, Lindenberger U, Li SC, Nyberg L (2010) Linking cognitive aging to alterations in dopamine neurotransmitter functioning: recent data and future avenues. Neurosci Biobehav Rev 34:670-677.

Barrick TR, Charlton RA, Clark CA, Markus HS (2010) White matter struc- 
tural decline in normal ageing: a prospective longitudinal study using tract-based spatial statistics. Neuroimage 51:565-577.

Bedard AC, Nichols S, Barbosa JA, Schachar R, Logan GD, Tannock R (2002) The development of selective inhibitory control across the life span. Dev Neuropsychol 21:93-111.

Behrens TE, Woolrich MW, Jenkinson M, Johansen-Berg H, Nunes RG, Clare S, Matthews PM, Brady JM, Smith SM (2003) Characterization and propagation of uncertainty in diffusion-weighted MR imaging. Magn Reson Med 50:1077-1088.

Behrens TE, Berg HJ, Jbabdi S, Rushworth MF, Woolrich MW (2007) Probabilistic diffusion tractography with multiple fibre orientations: what can we gain? Neuroimage 34:144-155.

Boorman ED, O'Shea J, Sebastian C, Rushworth MF, Johansen-Berg H (2007) Individual differences in white-matter microstructure reflect variation in functional connectivity during choice. Curr Biol 17:1426-1431.

Chikazoe J (2010) Localizing performance of go/no-go tasks to prefrontal cortical subregions. Curr Opin Psychiatry 23:267-272.

Coxon JP, Stinear CM, Byblow WD (2006) Intracortical inhibition during volitional inhibition of prepared action. J Neurophysiol 95:3371-3383.

Coxon JP, Stinear CM, Byblow WD (2007) Selective inhibition of movement. J Neurophysiol 97:2480-2489.

Coxon JP, Stinear CM, Byblow WD (2009) Stop and go: the neural basis of selective movement prevention. J Cogn Neurosci 21:1193-1203.

Coxon JP, Goble DJ, Van Impe A, De Vos J, Wenderoth N, Swinnen SP (2010) Reduced basal ganglia function when elderly switch between coordinated movement patterns. Cereb Cortex 20:2368-2379.

Dormont D, Ricciardi KG, Tand é D, Parain K, Menuel C, Galanaud D, Navarro S, Cornu P, Agid Y, Yelnik J (2004) Is the subthalamic nucleus hypointense on T2-weighted images? A correlation study using MR imaging and stereotactic atlas data. AJNR Am J Neuroradiol 25:1516-1523.

Duann JR, Ide JS, Luo X, Li CS (2009) Functional connectivity delineates distinct roles of the inferior frontal cortex and presupplementary motor area in stop signal inhibition. J Neurosci 29:10171-10179.

Fjell AM, Westlye LT, Amlien I, Espeseth T, Reinvang I, Raz N, Agartz I, Salat DH, Greve DN, Fischl B, Dale AM, Walhovd KB (2009) High consistency of regional cortical thinning in aging across multiple samples. Cereb Cortex 19:2001-2012.

Fleming SM, Thomas CL, Dolan RJ (2010) Overcoming status quo bias in the human brain. Proc Natl Acad Sci U S A 107:6005-6009.

Forstmann BU, Jahfari S, Scholte HS, Wolfensteller U, van den Wildenberg WP, Ridderinkhof KR (2008) Function and structure of the right inferior frontal cortex predict individual differences in response inhibition: a model-based approach. J Neurosci 28:9790-9796.

Forstmann BU, Anwander A, Schäfer A, Neumann J, Brown S, Wagenmakers EJ, Bogacz R, Turner R (2010) Cortico-striatal connections predict control over speed and accuracy in perceptual decision making. Proc Natl Acad Sci U S A 107:15916-15920.

Forstmann BU, Tittgemeyer M, Wagenmakers EJ, Derrfuss J, Imperati D, Brown S (2011) The speed-accuracy tradeoff in the elderly brain: a structural model-based approach. J Neurosci 31:17242-17249.

Forstmann BU, Keuken MC, Jahfari S, Bazin PL, Neumann J, Schäfer A, Anwander A, Turner R (2012) Cortico-subthalamic white matter tract strength predicts interindividual efficacy in stopping a motor response. Neuroimage 60:370-375.

Frank MJ (2006) Hold your horses: a dynamic computational role for the subthalamic nucleus in decision making. Neural Netw 19:1120-1136.

Giovannelli F, Borgheresi A, Balestrieri F, Zaccara G, Viggiano MP, Cincotta M, Ziemann U (2009) Modulation of interhemispheric inhibition by volitional motor activity: an ipsilateral silent period study. J Physiol 587:5393-5410.

Haacke EM, Mittal S, Wu Z, Neelavalli J, Cheng YC (2009) Susceptibilityweighted imaging: technical aspects and clinical applications, part 1. AJNR Am J Neuroradiol 30:19-30.

Hasher L, Zacks RT (1988) Working memory, comprehension, and aging: a review and a new view. In: The psychology of learning and motivation (Bower GH, ed), pp 193-225. New York: Academic.

Hedden T, Gabrieli JD (2004) Insights into the ageing mind: a view from cognitive neuroscience. Nat Rev Neurosci 5:87-96.

Hikosaka O, Isoda M (2010) Switching from automatic to controlled behavior: cortico-basal ganglia mechanisms. Trends Cogn Sci 14:154-161.

Inase M, Tokuno H, Nambu A, Akazawa T, Takada M (1999) Corticostriatal and corticosubthalamic input zones from the presupplementary mo- tor area in the macaque monkey: comparison with the input zones from the supplementary motor area. Brain Res 833:191-201.

Isoda M, Hikosaka O (2007) Switching from automatic to controlled action by monkey medial frontal cortex. Nat Neurosci 10:240-248.

Isoda M, Hikosaka O (2008) Role for subthalamic nucleus neurons in switching from automatic to controlled eye movement. J Neurosci 28:7209-7218.

Johansen-Berg H (2010) Behavioural relevance of variation in white matter microstructure. Curr Opin Neurol 23:351-358.

Johansen-Berg H, Behrens TE, Robson MD, Drobnjak I, Rushworth MF, Brady JM, Smith SM, Higham DJ, Matthews PM (2004) Changes in connectivity profiles define functionally distinct regions in human medial frontal cortex. Proc Natl Acad Sci U S A 101:13335-13340.

Kennedy KM, Raz N (2009) Aging white matter and cognition: differential effects of regional variations in diffusion properties on memory, executive functions, and speed. Neuropsychologia 47:916-927.

Kenner NM, Mumford JA, Hommer RE, Skup M, Leibenluft E, Poldrack RA (2010) Inhibitory motor control in response stopping and response switching. J Neurosci 30:8512-8518.

King AV, Linke J, Gass A, Hennerici MG, Tost H, Poupon C, Wessa M (2012) Microstructure of a three-way anatomical network predicts individual differences in response inhibition: a tractography study. Neuroimage 59:1949-1959.

Kramer AF, Humphrey DG, Larish JF, Logan GD, Strayer DL (1994) Aging and inhibition: beyond a unitary view of inhibitory processing in attention. Psychol Aging 9:491-512.

Levy BJ, Wagner AD (2011) Cognitive control and right ventrolateral prefrontal cortex: reflexive reorienting, motor inhibition, and action updating. Ann N Y Acad Sci 1224:40-62.

Lucerna S, Salpietro FM, Alafaci C, Tomasello F (2002) In vivo atlas of deep brain structures. Berlin: Springer.

Lustig C, Hasher L, Zacks RT (2007) Inhibitory deficit theory: recent developments in a "new view." In: The place of inhibition in cognition (Gorfein DS, MacLeod CM, eds), pp 145-162. Washington, DC: American Psychological Association.

Madsen KS, Baar é WF, Vestergaard M, Skimminge A, Ejersbo LR, Ramsøy TZ, Gerlach C, Akeson P, Paulson OB, Jernigan TL (2010) Response inhibition is associated with white matter microstructure in children. Neuropsychologia 48:854-862.

Mars RB, Klein MC, Neubert FX, Olivier E, Buch ER, Boorman ED, Rushworth MF (2009) Short-latency influence of medial frontal cortex on primary motor cortex during action selection under conflict. J Neurosci 29:6926-6931

Munakata Y, Herd SA, Chatham CH, Depue BE, Banich MT, O'Reilly RC (2011) A unified framework for inhibitory control. Trends Cogn Sci 15:453-459.

Nambu A, Tokuno H, Takada M (2002) Functional significance of the cortico-subthalamo-pallidal "hyperdirect" pathway. Neurosci Res 43:111-117.

Neubert FX, Mars RB, Buch ER, Olivier E, Rushworth MF (2010) Cortical and subcortical interactions during action reprogramming and their related white matter pathways. Proc Natl Acad Sci U S A 107:13240-13245.

O'Sullivan M, Jones DK, Summers PE, Morris RG, Williams SC, Markus HS (2001) Evidence for cortical "disconnection" as a mechanism of agerelated cognitive decline. Neurology 57:632-638.

Penke L, Muñoz Maniega S, Murray C, Gow AJ, Hernández MC, Clayden JD, Starr JM, Wardlaw JM, Bastin ME, Deary IJ (2010) A general factor of brain white matter integrity predicts information processing speed in healthy older people. J Neurosci 30:7569-7574.

Ray NJ, Jenkinson N, Brittain J, Holland P, Joint C, Nandi D, Bain PG, Yousif N, Green A, Stein JS, Aziz TZ (2009) The role of the subthalamic nucleus in response inhibition: Evidence from deep brain stimulation for Parkinson's disease. Neuropsychologia 47:2828-2834.

Sharp DJ, Bonnelle V, De Boissezon X, Beckmann CF, James SG, Patel MC, Mehta MA (2010) Distinct frontal systems for response inhibition, attentional capture, and error processing. Proc Natl Acad Sci U S A 107:6106-6111.

Smith SM, Nichols TE (2009) Threshold-free cluster enhancement: addressing problems of smoothing, threshold dependence and localisation in cluster inference. Neuroimage 44:83-98.

Smith SM, Jenkinson M, Johansen-Berg H, Rueckert D, Nichols TE, Mackay CE, Watkins KE, Ciccarelli O, Cader MZ, Matthews PM, Behrens TE 
(2006) Tract-based spatial statistics: voxelwise analysis of multi-subject diffusion data. Neuroimage 31:1487-1505.

Sullivan EV, Pfefferbaum A (2006) Diffusion tensor imaging and aging. Neurosci Biobehav Rev 30:749-761.

Talelli P, Ewas A, Waddingham W, Rothwell JC, Ward NS (2008) Neural correlates of age-related changes in cortical neurophysiology. Neuroimage 40:1772-1781.

Tzourio-Mazoyer N, Landeau B, Papathanassiou D, Crivello F, Etard O, Delcroix N, Mazoyer B, Joliot M (2002) Automated anatomical labeling of activations in SPM using a macroscopic anatomical parcellation of the MNI MRI single-subject brain. Neuroimage 15:273-289.

Van Impe A, Coxon JP, Goble DJ, Doumas M, Swinnen SP (2011) White matter fractional anisotropy predicts balance performance in older adults. Neurobiol Aging. Advance online publication. Retrieved May 10, 2012. doi:10.1016/j.neurobiolaging.2011.06.013.

Verbruggen F, Logan GD (2009) Models of response inhibition in the stopsignal and stop-change paradigms. Neurosci Biobehav Rev 33:647-661.
Vernooij MW, Ikram MA, Vrooman HA, Wielopolski PA, Krestin GP, Hofman A, Niessen WJ, Van der Lugt A, Breteler MM (2009) White matter microstructural integrity and cognitive function in a general elderly population. Arch Gen Psychiatry 66:545-553.

Walker HC, Watts RL, Schrandt CJ, Huang H, Guthrie SL, Guthrie BL, Montgomery EB Jr (2011) Activation of subthalamic neurons by contralateral subthalamic deep brain stimulation in Parkinson disease. J Neurophysiol 105:1112-1121.

Williams BR, Ponesse JS, Schachar RJ, Logan GD, Tannock R (1999) Development of inhibitory control across the life span. Dev Psychol 35:205-213.

Zahr NM, Rohlfing T, Pfefferbaum A, Sullivan EV (2009) Problem solving, working memory, and motor correlates of association and commissural fiber bundles in normal aging: a quantitative fiber tracking study. Neuroimage 44:1050-1062.

Zandbelt BB, Vink M (2010) On the role of the striatum in response inhibition. PLoS One 5:e13848. 